\title{
Quantitative Analysis of Iron(II) Solution by Photothermal Deflection Spectrometry
}

\author{
Akinori Uejima*, Masanobu Habiro*, Fumio Itoga*, Yoshinori Sugitani* \\ and Kenji KATo**
}

*Institute of Chemistry, University of Tsukuba, Sakura-mura, Ibaraki 305

**National Chemical Laboratory for Industry, Yatabe-machi, Ibaraki 305

Keywords Photothermal deflection spectrometry, iron(II) determination, 1, 10-phenanthroline

Photothermal deflection spectroscopy (PDS) is the measurement of the light absorptions by a sample by optical detection of the refractive index gradient formed in or near the sample. It is based on the "mirage effect" which was first reported by Boccara et al. It is closely related to photoacoustic spectroscopy which also observes the photothermal process and detects the generation of heat in the light absorbing material.

In the study of photoacoustic and correlation photoacoustic spectroscopy on various kind of samples using a photoacoustic cell with a microphone as a detector, there has been a problem, especially when working on fluid sample, that the fluid sample resonates in the photoacoustic cell giving a strong wave form signal superposed on the tail of impulse response curve to be observed. This effect limits the measurement of the spectra at modulation frequencies higher than the resonating frequency. To overcome the limitation, the photothermal deflection method in place of acoustic detection is being attempted to be used in our measuring system. This method is considered to be resonance free and at the same time to be a very high sensitive detection method when applied

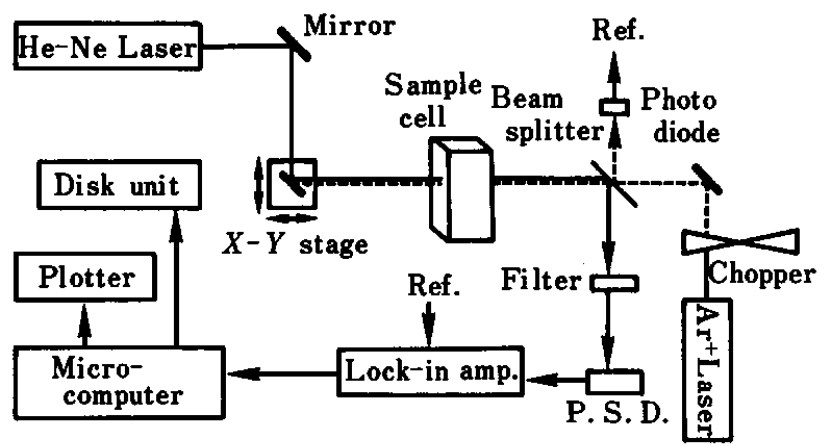

Fig. I Blockdiagram showing the experimental setup for photothermal deflection spectrometry. P.S.D., position sensitive detector.

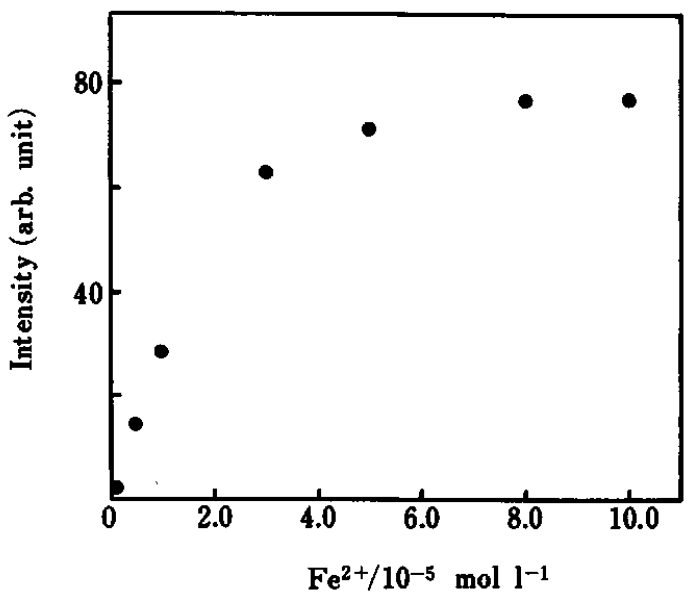

Fig. 2(A) Plots of signal intensity versus Fe(II) concentration in the range $10^{-4}-10^{-6} \mathrm{~mol} / 1$.

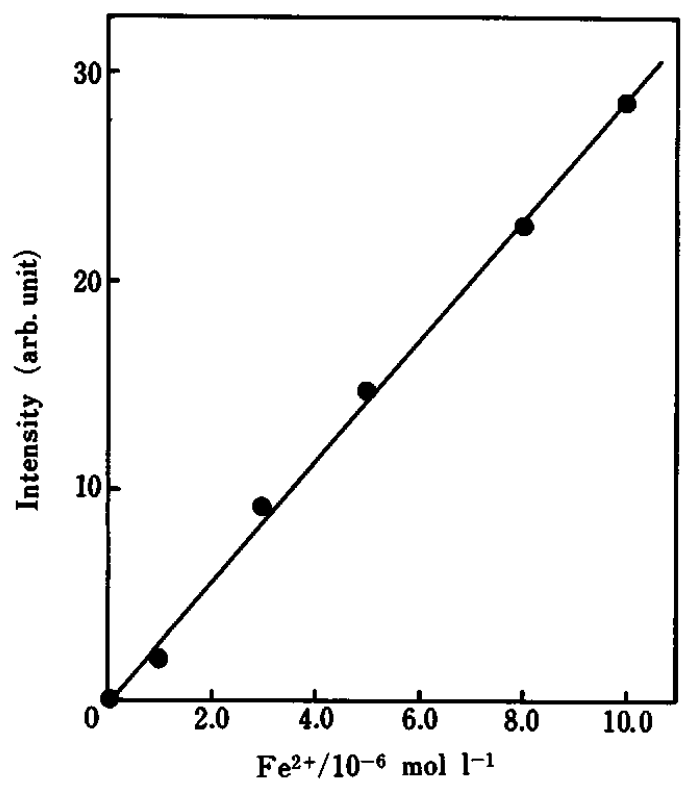

Fig. 2(B) Plots of signal intensity versus $\mathrm{Fe}(\mathrm{II})$ concentration showing the linear relation in the concentration range $1-10 \times 10^{-6} \mathrm{~mol} / 1$. 
to the measurement of dilute solution ${ }^{2-4}$. In the present study, the PDS method using paralleled pump and probe beams has been applied to the quantitative analysis of $\mathrm{Fe}(\mathrm{II})$ solution.

Water solutions of $\mathrm{Fe}(\mathrm{II})-1,10$-phenanthroline with the concentration ranging from $10^{-6}-10^{-4} \mathrm{~mol} / 1$ have been prepared. Preparation of these solutions were made after the standard procedure found in literature. ${ }^{5}$ Figure 1 shows the experimental setup for the measuring system of photothermal deflection method. The $488.0 \mathrm{~nm}$ beam (150 $\mathrm{mW}$ ) of argon ion laser(NEC, GLG3300) was used as a pumping light source. The beam was chopped by a mechanical chopper at $15 \mathrm{~Hz}$ and was irradiated to the sample solution in the quartz cell having the dimension $20 \mathrm{~mm}(\mathrm{~L}) \times 10 \mathrm{~mm}(\mathrm{~W})$. The probe beam of $632.8 \mathrm{~nm}(0.5 \mathrm{~mW})$ of $\mathrm{He}-\mathrm{Ne}$ laser(NEC, GLC5090) was also irradiated to the sample solution with its beamline parallel to the pumping beam in the opposite direction against the pumping beam. The beam diameter of the pump and probe beam was $1.5 \mathrm{~mm} \phi$ and $0.7 \mathrm{~mm} \phi$, respectively. The separation of the both beams was about $0.3 \mathrm{~mm}$ from center to center. The separation of the beams was finely adjusted by moving the mirror on a $X-Y$ stage. Part of the deflected beam was reflected by a halfmirror and was lead onto a position sensitive detector(PSD) by which the displacement of the beam spot was converted into voltage.

Figure 2(A) shows the relation between the concentration of the sample solution and the signal intensity which is directly proportional to the displacement of the beam spot. There was a distinct saturation effect in the concentration range $1-10 \times 10^{-5} \mathrm{~mol} / 1$ which was commonly used in the colorimetric determination of ferrous ion. There seemed, however, to be a linear relation in the lower concentration range. Figure 2(B) distinctly shows the linear relation of the signal intensity versus iron concentration in the range $1-10 \times$ $10^{-6} \mathrm{~mol} / \mathrm{l}$. In the plots of the intensity data in Fig. 2(B) the contribution from the blank solution was subtracted so as to give zero intensity for zero concentration. This preliminary results show that the PDS method can be applied to the quantitative analysis of metal-chelate solution in lower concentration range than in the ordinary colorimetric method. It should be noted, however, that Miyaishi et al. have reported more sensitive determination $\left(10^{-8}\right.$ mol/l level for $\mathrm{Fe}(\mathrm{II})$ complex) by thermal lens spectrophotometry. ${ }^{6}$ Higher sensitivity would be obtained by some improvements such as the use of stronger pumping source, use of organic solvents in place of water, and so on.

\section{References}

1. A. C. Boccara, D. Fournier and J. Badoz: Appl. Phys. Lett., 36, 130 (1980).

2. D. Fournier, A. C. Boccara, N. M. Amer and R. Gerlach, Appl. Phys. Lett., 37, 519 (1980).

3. J. A. Sell, Appl. Opt., 23, 1586 (1984).

4. S. W. Kizirnis, R. J. Brecha, B. N. Ganguly, L. P. Goss and R. Gupta, Appl. Opt., 23, 3873 (1984).

5. For instance, K. Nagashima and I. Tomita: "Bunseki Kagaku", p. 283, Shokabo, Tokyo (1985).

6. K. Miyaishi, T. Imasaka and N. Ishibashi, Anal. Chem., 54, 2039 (1982).

(Received April 30, 1986)

(Accepted May 14, 1986) 